\title{
On Avoiding Spare Aborts in Transactional Memory
}

\author{
Idit Keidar \\ Dmitri Perelman \\ Dept. of Electrical Engineering \\ Technion, Haifa 32000, Israel \\ idish@ee.technion.ac.il, dima39@tx.technion.ac.il
}

\begin{abstract}
This paper takes a step toward developing a theory for understanding aborts in transactional memory systems (TMs). Existing TMs may abort many transactions that could, in fact, commit without violating correctness. We call such unnecessary aborts spare aborts. We classify what kinds of spare aborts can be eliminated, and which cannot. We further study what kinds of spare aborts can be avoided efficiently. Specifically, we show that some unnecessary aborts cannot be avoided, and that there is an inherent tradeoff between the overhead of a TM and the extent to which it reduces the number of spare aborts. We also present an efficient example TM algorithm that avoids certain kinds of spare aborts, and analyze its properties and performance.
\end{abstract}




\section{Introduction}

The emergence of multi-core architectures raises the problem of efficient synchronization in multithreaded programs. Conventional locking solutions introduce a host of well-known problems: coarse-grained locks are not scalable, while fine-grained locks are error-prone and hard to design. Transactional memory [10, 15] has gained popularity in recent years as a new synchronization abstraction for multithreaded systems, which has the potential to overcome the pitfalls of traditional locking schemes. A transactional memory toolkit, or $T M$ for short, allows threads to bundle multiple operations on memory objects into one transaction. Similarly to database transactions [16], transactions are executed atomically: either all of the transaction's operations appear to take effect simultaneously (in this case, we say that the transaction commits), or none of transaction's operations are seen (in this case, we say that transaction aborts). We formally define the model and correctness criterion in Section 3.

A transaction's abort may be initiated by a programmer or may be the result of a TM decision. In the latter case, we say that the transaction is forcefully aborted by the TM. For example, when one transaction reads some object A and then writes to some object B, while another transaction reads the old value of B and then attempts to write A, one of the transactions must be aborted in order to ensure atomicity. Most existing TMs perform unnecessary (spare) aborts, i.e., aborts of transactions that could have committed without violating correctness; see Section 2. Spare aborts have several drawbacks: work done by the aborted transaction is lost, computer resources are wasted, and the overall throughput decreases. Moreover, after the aborted transactions restart, they may conflict again, leading to livelock and degrading performance even further.

The aim of this paper is to advance the theoretical understanding of TM aborts, by studying what kinds of spare aborts can or cannot be eliminated, and what kinds of spare aborts can or cannot be avoided efficiently. Specifically, we show that some unnecessary aborts cannot be avoided, and that there is an inherent tradeoff between the overhead of a TM and the extent to which it refrains from spare aborts.

Previous works introduced two related notions: commit-abort ratio [6] and permissiveness [7]. The latter stipulates that in runs that do not violate correctness, no aborts should happen. However, while shedding insight on the inherent limitations of online TMs, these notions do not provide an interesting yardstick for comparing TMs. This is because under these measures, all online TMs inherently perform poorly for some worst-case workloads, as we show in Section 4.

In Section 5, we then define measures of spare aborts that are appropriate for online TMs. Intuitively, our strict online permissiveness property allows a TM to abort some transaction only if not aborting any transaction would violate correctness. Unlike ealier notions, strict online permissiveness does not prevent the TM from taking an action that might lead to an abort in the future. Thus, the information available to the $\mathrm{TM}$ at every given moment suffices to implement strict online permissiveness. Clearly, this property depends on the correctness criterion the TM needs to satisfy. In this paper, we consider opacity [8] or slight variants thereof (see Section 3). In this context, strict online permissiveness prohibits aborting a transaction whenever the execution history is equivalent to some sequential one. We prove that strict online permissiveness cannot be satisfied efficiently by showing a reduction from the NP-hard view serializability [13] problem. We then define a more relaxed property, online permissiveness, which allows the TM to abort transactions if otherwise it would have to change the serialization order between already committed transactions. We show that online permissiveness also has inherent costs — it cannot be satisfied by a TM using invisible reads. Moreover, the information about a read should be exposed in shared memory immediately after the read operation returns.

In Section 6, we show a polynomial time TM protocol satisfying online permissiveness. The protocol 
maintains a precedence graph of transactions and keeps it acyclic. Unfortunately, we show that the graph must contain some committed transactions. But without removing any committed transactions, detecting cycles in the precedence graph would be impractical as it would induce a high runtime complexity. Hence, we define precise garbage collection rules for removing transactions from the graph. Even so, a naïve traversal of the graph would be costly; we further introduce optimization techniques that decrease the number of nodes traversed during the acyclity check.

Finally, we note that our goal is not to build a better TM, but rather to understand what can and what cannot be achieved, and at what cost. Future work may further explore the practical aspects of the complexity vs. spare-aborts tradeoffs; our conclusions appear in Section 7.

\section{Related Work}

Most existing TM implementations, e.g., $[9,5,4,3]$ abort one transaction whenever two overlapping transactions access the same object and at least one access is a write. While easy to implement, this approach may lead to high abort rates, especially in situations with long-running transactions and contended shared objects. Aydonat and Abdelrahman [2] referred to this problem and proposed a solution based on a conflict serializability graph and multi-versioned objects in order to reduce the number of unnecessary aborts. However, their solution still induces spare aborts, and does not characterize exactly when such aborts are avoided. Moreover, they implement a stricter correctness criterion than opacity, which inherently requires more aborts. Riegel et al. [14] looked at the problem of spare aborts from a different angle, and introduced weaker correctness criteria, which allow TMs to reduce the number of aborts.

Napper and Alvisi [12] described a serializable TM, based upon multi-versioned objects, which used cycle detection in the precedence graph when validating the correctness criterion. The focus of the paper was providing a lock-free solution. The authors did not refer to the aspect of spare aborts and, in fact, their TM did lead to spare aborts due to a limitation on write operation, which had to insert the new version after the latest one. In addition, Napper and Alvisi did not refer to the problems of garbage collection and computational complexity of operations.

Gramoli et al. [6] referred to the problem of spare aborts and introduced the notion of commit-abort ratio, which is the ratio between the number of committed transactions and the overall number of transactions in the run. Clearly, the commit-abort ratio depends on the choice of the transaction that should be aborted in case of a conflict. This decision is the prerogative of a contention manager [9]. Attiya et al. [1] showed a $\Omega(s)$ lower bound for the competitive ratio for transactions' makespan of any online deterministic contention manager, where $s$ is the number of shared objects. Their proof, however, does not apply to our model, because it is based upon the assumption that whenever multiple transactions need exclusive access to the same shared object, only one of these transactions may continue, while others should be immediately aborted. In contrast, our model allows the TM to postpone the decision regarding which transaction should be aborted till the commit, thus introducing additional knowledge and improving the competitive ratio. In this paper, we show that every TM is $\Omega(L)$ competitive in terms of commit-abort ratio, where $L$ is the number of live transactions in the system. This result suggests that it is not interesting to compare (online) TMs by their commit-abort ratio, as the distance from the optimal result turns out to be an artifact of the workload rather than the algorithm, and every TM has a workload on which it performs poorly by this measure.

Input acceptance is also a notion presented by Gramoli et al. [6] - a TM accepts a certain input pattern (sequence of invocations) if it commits all of its transactions. The authors compared different TMs according to their input acceptance patterns. Guerraoui et al. [7] introduced the related notion of $\pi$-permissiveness. Informally, a TM satisfies $\pi$-permissiveness for a correctness criterion $\pi$, if every history that does not 
violate $\pi$ is accepted by the TM. Thus, $\pi$-permissiveness can be seen as optimal input acceptance. However, Guerraoui et al. focused on a model with single-version objects, and their correctness criterion was based upon conflict serializability, which is stronger than opacity and thus allows more aborts. They ruled out the idea of ensuring permissiveness deterministically, and instead provide a randomized solution, which is always correct and avoids spare aborts with some positive probability. In contrast, we do not limit the model to include single-version objects only, our correctness criterion is a generalization of opacity [8], and we focus on deterministic guarantees. Although permissiveness does not try to regulate the decisions of the contention manager, we show that no online TM may achieve permissiveness. Intuitively, this results from the freedom of choice for returning the object value during the read operation - returning the wrong value might cause an abort in subsequent operations, which is avoided by a clairvoyant (offline) algorithm.

\section{Preliminaries and System Model}

Transactions. Our definition of Transactional Memory (TM) is based on [8]. A TM allows threads to run transactions. Transactions perform operations on shared objects. The objects considered in this paper are read/write registers. The status of a transaction may be either live, aborted, or committed. A transaction can perform operations as long as it is live. Each transaction has a unique identifier (id). Retrying an aborted transaction is interpreted as creating a new transaction with a new id. The maximal possible number of live transactions is $L$.

The API of the TM includes the following operations. The operation startTransaction() returns the id of a newly created transacton. The status of a newly created transaction is always live. When $T_{i}$ is live, it can invoke the following operations: $\operatorname{read}\left(T_{i}, o\right)$, which returns the value of register $o$, and write $\left(T_{i}, o, v\right)$, which writes value $v$ to register $o$. For the sake of simplicity, we assume that the values written to the registers are unique. When $T_{i}$ wishes to terminate, it invokes operation $\operatorname{try} \operatorname{Commit}\left(T_{i}\right)$ or $\operatorname{try} \operatorname{Abort}\left(T_{i}\right)$. If $\operatorname{try} \operatorname{Commit}\left(T_{i}\right)$ returns $C_{i}$, the status of $T_{i}$ changes to committed, while $\operatorname{try} A b \operatorname{brt}\left(T_{i}\right)$ always returns $A_{i}$, indicating that $T_{i}$ is aborted. The abort value $A_{i}$ may also be returned as a response to read, write or tryCommit invocations, in which case we say that the TM forcefully aborts transaction $T_{i}$. If the TM forcefully aborts transaction $T_{j}$ as a result of another transaction's operation, then the returned value of the subsequent operation of $T_{j}$ will be $A_{j}$. The read-set and the write-set of $T_{i}$ are denoted as $\operatorname{read}\left(T_{i}\right)$ and write $\left(T_{i}\right)$ respectively, and are not known in advance.

The calls to the TM are blocking - the invoking thread waits for a response before invoking more operations. We assume that TM operations issued by different threads are executed atomically. This allows us to neglect issues related to overlapping operation executions, which are not the focus of this paper; in practice, such atomicity can be implemented using locks or well-known lock-free solutions, e.g., [5]. Note, however, that transactions may overlap.

The TM guarantees that each operation invocation eventually gets a response, even if all other threads are sleeping. This limits the TM's behavior upon operation invocation, so that it may either return an operation response, or abort a transaction, but cannot wait for other transactions to invoke operations.

Transaction histories. A transaction history is the sequence of operations issued by transactions in a given TM execution, ordered by the time at which they are issued (in the rest of the paper we use the notion of run as a synonim to a transaction history). Two histories $H_{1}$ and $H_{2}$ are equivalent if they contain the same transactions and each transaction $T_{i}$ issues the same operations in the same order with the same responses in both. A history $H$ is complete if it does not contain live transactions. If history $H$ is not complete, we may build from it a complete history Complete $(H)$ by adding an abort operation for every live transaction. 
We define committed $(H)$ to be the subsequence of $H$ consisting of all the operations of all the committed transactions in $H$.

The real-time order on transactions is as follows: if the first event of transaction $T_{i}$ is issued after the last response of transaction $T_{j}$ in $H$, then $T_{j} \prec_{H} T_{i}$. Transactions $T_{i}$ and $T_{j}$ are concurrent if neither $T_{j} \prec_{H} T_{i}$, nor $T_{i} \prec_{H} T_{j}$. A history $S$ is sequential if it has no concurrent transactions. A sequential history $S$ is legal if it respects the sequential specification of each object accessed in $S$. Transaction $T_{i}$ is legal in $S$ if the largest subsequence $S^{\prime}$ of $S$, such that, for every transaction $T_{k} \in S^{\prime}$, either (1) $k=i$, or (2) $T_{k}$ is committed and $T_{k} \prec_{S} T_{i}$, is a legal history.

Correctness. Our correctness criterion resembles the opacity condition of Guerraoui and Kapalka [8]. Let $\Gamma(H)$ be a partial order on transactions. A TM satisfies $\Gamma$-opacity if for every history $H$ generated by the TM there exists a sequential history $S$, s.t.:

- $S$ is equivalent to Complete $(H)$.

- Every transaction $T_{i} \in S$ is legal in $S$.

- If $\left(T_{i}, T_{j}\right) \in \Gamma(H)$, then $T_{i} \prec_{S} T_{j}$.

When $\Gamma(H)$ includes all the ordered pairs of non-concurrent transactions in $H$, the history $S$ should preserve the real-time order of $H$. On the other hand, when $\Gamma$ is empty, the correctness criterion is a serializability with consideration of aborted transactions. The use of $\Gamma$ makes it possible to require transactional ordering that lies between serializability and strict serializability according to any arbitrary rule (e.g., Riegel et al. [14] considered demanding real-time order only from transactions belonging to the same thread). We define a more general criterion in order to broaden the scope of our results. In the rest of this paper, we will assume that $\Gamma(H)$ is a subset of the real-time order on transactions, unless stated otherwise.

We should note that our notion of $\Gamma$-opacity is somewhat stronger than the original one defined by Guerraoui and Kapalka [8], in that it is a safety property (i.e., prefix-closed). Since the set of histories of every TM is prefix closed, every TM satisfying the original (weaker) property also satisfies the (stronger) version we define. Moreover, the notion of opacity-permissiveness does not make sense in the context of the former because no TM may generate opaque histories that have non-opaque prefixes. Indeed, the paper that defines permissiveness [7] uses an even stronger notion of opacity, which is in fact prefix-closed.

\section{Limitations of Previous Measures}

\subsection{Commit-Abort Ratio}

The commit-abort ratio $(\tau)$ [6] is the ratio between the number of committed transactions and the overall number of transactions in the history. Unfortunately, no online TM may guarantee optimal commit-abort ratio. Recall that $L$ is the number of live transactions. We show that every TM is $\Omega(L)$ competitive in terms of its commit-abort ratio.

We use the style of [14] to depict transactional runs. Objects are represented as horizontal lines $o_{1}, o_{2}$, etc. Transactions are drawn as polylines with circles corresponding to accesses to the objects. Filled circles indicate writes, and empty circles indicate reads. Commit is indicated by the letter $\mathbf{C}$, and abort by the letter A. If the TM implements the access to the object as if it had appeared in past, the dashed arc indicates the point in time at which the access to the object appears according to the TM serialization. 


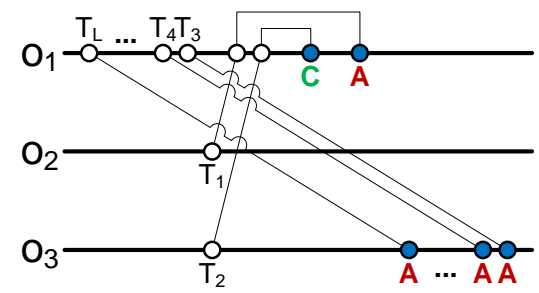

(a) Run $r_{1}: T_{2}$ commits, all other transactions abort: $\tau=\frac{1}{L}$

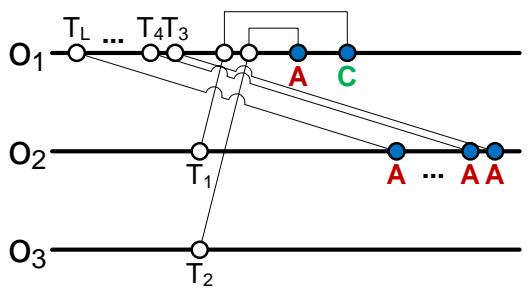

(b) Run $r_{2}: T_{1}$ commits, all other transactions abort: $\tau=\frac{1}{L}$

Figure 1: No online TM may know whether to abort $T_{1}$ or $T_{2}$ in order to obtain an optimal commit-abort ratio.

Lemma 1. Every TM is $\Omega(L)$ competitive in terms of its commit-abort ratio.

Proof. Consider the scenarios depicted in Figure 1. The runs are indistinguishable until the time when $T_{L}$ tries to commit. Transactions $T_{1}$ and $T_{2}$ cannot both commit because both write $o_{1}$ after reading its previous value. In run $r_{1}$ (Figure 1(a)), the TM commits $T_{2}$, then $T_{1}$ aborts and then the transactions $T_{3} \cdots T_{L}$ try to write to $o_{3}$ and must be aborted because they conflict with $T_{2}$, resulting in $\tau=\frac{1}{L}$. In run $r_{2}$ (Figure 1(b)), the TM aborts $T_{2}, T_{1}$ commits and then the transactions $T_{3} \cdots T_{L}$ try to write to $o_{2}$ and therefore must be aborted, resulting again in $\tau=\frac{1}{L}$. The optimal offline TM in these cases would abort only one transaction, yielding $\tau=\frac{L-1}{L}$. The online TM, however, cannot distinguish between $r_{1}$ and $r_{2}$ at the moment it should decide whether to abort $T_{1}$ or $T_{2}$, hence the competitive ratio is $\Omega(L)$.

\subsection{Permissiveness}

Since requiring an optimal commit-abort ratio is too restrictive, we consider a weaker notion that limits aborts only in runs where none are necessary: a TM provides permissiveness [7] if it accepts every history satisfying $\Gamma$-opacity. Gramoli et al. showed that existing TM implementations do not accept all inputs they could have, and hence are not permissive. We show that this is an inherent limitation.

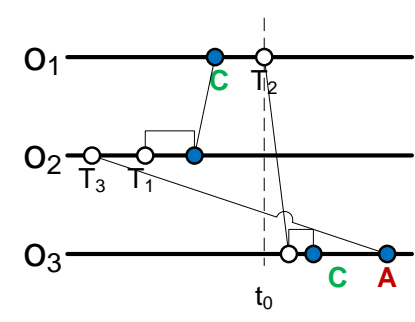

(a) Run $r_{1}: T_{2}$ reads the value $v_{1}$

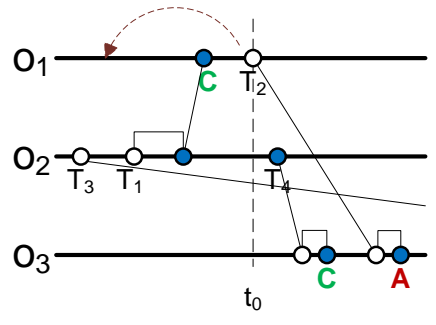

(b) Run $r_{2}: T_{2}$ reads the value $v_{0}$

Figure 2: At time $t_{0}$, no online TM knows which value should be returned to $T_{2}$ when reading $o_{1}$ in order to allow for commit in the future.

The formal impossibility illustrated in Figure 2 is captured in the following lemma:

Lemma 2. For any $\Gamma$, there is no online TM implementation providing $\Gamma$-opacity-permissiveness. 
Proof. Consider the scenario depicted in Figure 2. All the objects have initial values, $v_{0}$. All the transactions start at the same time, $t_{i}$, and are therefore not ordered according to the real-time order, thus the third condition of our correctness criterion holds for any $\Gamma$.

$T_{1}$ writes values $v_{1}$ to $o_{2}$ and $o_{1}$. At time $t_{0}$, there is a read operation of $T_{2}$ and the TM should decide what value should be returned. In general, the TM has four possibilities: (1) return $v_{1}$, (2) return $v_{0}$, (3) return some value $v^{\prime}$ different from $v_{0}$ and $v_{1}$, and (4) abort $T_{2}$. If the TM chooses to abort, then opacitypermissiveness is violated and we are done. (3) is not possible, for returning such a value would produce a history, for which any equivalent sequential history $S$ would violate the sequential specification of $o_{1}$ and thus would not be legal.

Consider case (1): the TM returns $v_{1}$ for $T_{2}$ at time $t_{0}$. This serializes $T_{2}$ after $T_{1}$. Consider run $r_{1}$ depicted in Figure 2(a), where $T_{3}$ tries to write to $o_{3}$ and commit. In this run, the TM has to forcefully abort $T_{3}$, because not doing so would produce a history $H$ with no equivalent sequential history: $T_{1} \prec T_{2} \prec T_{3} \prec$ $T_{1}$. However, if $T_{2}$ would read $v_{0}$ in run $r_{1}$, then $T_{2}, T_{1}$ and $T_{3}$ would be legal, and no transaction would have to be forcefully aborted. So $\Gamma$-opacity-permissiveness is violated.

In case (2), the TM returns $v_{0}$ for transaction $T_{2}$ at time $t_{0}$, serializing $T_{2}$ before $T_{1}$. Consider run $r_{2}$ depicted in Figure 2(b). Transaction $T_{4}$ writes to $o_{2}$, and afterwards reads and writes to $o_{3}$. Transaction $T_{4}$ has to be serialized after $T_{1}$, because $T_{1}$ has read $v_{0}$ from $o_{2}$. When $T_{2}$ will try to write to $o_{3}$ and commit, the TM will have to forcefully abort some transaction, because not doing so would produce a history with no equivalent sequential history: $T_{2} \prec T_{1} \prec T_{4} \prec T_{2}$. But if $T_{2}$ would read $v_{1}$ in run $r_{2}$, then no transaction would have to be forcefully aborted. So again, $\Gamma$-opacity-permissiveness is violated.

Runs $r_{1}$ and $r_{2}$ are indistinguishable to the TM at time $t_{0}$. Therefore, no online TM can accept both of the patterns, while an offline TM can accept both of them.

\section{Online permissiveness: limitations and costs}

\subsection{Strict Online Opacity-Permissiveness}

We next define a property that prohibits unnecessary aborts, and yet is possible to implement.

Definition 1. A TM satisfies strict online $\Gamma$-opacity-permissiveness if the TM forcefully aborts a set $S$ of live transactions only when aborting any subset $S^{\prime} \subset S$ of transactions violates $\Gamma$-opacity for the given $\Gamma$.

Note that this property does not define which transaction should be aborted if abort happens, and does not prohibit returning a value that will cause aborts in the future. For example, in the scenarios depicted in Figure 2 , at time $t_{0}$, a TM satisfying this property may return either value, even though this might cause an abort in the future.

An algorithm satisfying strict online opacity-permissiveness should be able to detect whether returning a given value creates a history satisfying $\Gamma$-opacity. We show that this cannot be detected efficiently. To this end, we recall a well-known result about checking the serializability of the given history, which was proven by Papadimitriou [13].

Given history $H$, the augmented history $\bar{H}$ is the history, which is identical to $H$, except two additional transactions: $T_{\text {init }}$ that initializes all variables without reading any, and $T_{\text {read }}$ that is the last transaction of $\bar{H}$, reading all variables without changing them. The set of live transactions in $H$ is defined in the following way: (1) $T_{\text {read }}$ is live in $H$, (2) If for some live transaction $T_{j}, T_{j}$ reads a variable from $T_{i}$, then $T_{i}$ is also live in $H$. Note that aborted transaction cannot be live according to this definition (no transaction may read the values written by the aborted one). Transaction is dead if it is not live. Two histories $H$ and $H^{\prime}$ are view 
equivalent if and only if (1) they have the same sets of live transactions and (2) $T_{i}$ reads from $T_{j}$ in $H$ if and only if $T_{i}$ reads from $T_{j}$ in $H^{\prime}$. History $H$ is view serializable, if for every prefix $H^{\prime}$ of $H, \operatorname{complete}\left(H^{\prime}\right)$ is view equivalent to some serial history $S$.

Theorem 1 (Papadimitriou). Testing whether the history $H$ is view-serializable is NP-complete in the size of the history, even if $H$ has no dead transactions.

Lemma 3. For any $\Gamma$, detecting whether the history $H$ satisfies $\Gamma$-opacity is NP-complete in the size of the history.

Proof. We will show a reduction from the NP-complete problem of detecting view-seializability of history $H$ without dead transactions to the problem of detecting whether some history $H^{\prime}$ satisfies $\Gamma$-opacity. Consider history $H$ with no dead transactions. In the absence of aborted transactions, the definition of view serializability differs from the definition of opacity only in the fact that opacity refers to the partial order $\Gamma$, which is a subset of a real-time order. We construct history $H^{\prime}$, which is identical to history $H$ except the following addition: for each $T_{i}$ in $H$, we add $\operatorname{start}\left(T_{i}\right)$ at the beginning of $H^{\prime}$. We will show that $H$ is view serializable if and only if $H^{\prime}$ satisfies $\Gamma$-opacity.

$H$ is view serializable if and only if there exists a legal sequential history $S$, which is view equivalent to Complete $(H)$. All the transactions in $H^{\prime}$ are concurrent $\left(\operatorname{start}\left(T_{i}\right)\right.$ follows before any other event for every $T_{i}$ ), therefore the third condition of $\Gamma$-opacity vacuously holds for any $\Gamma$. In the absence of aborts in $H^{\prime}$, $H^{\prime}$ satisfies $\Gamma$-opacity if and only if there exists a legal sequential history $S^{\prime}$, so that every transaction in $H^{\prime}$ issues the same invocation events and receives the same response events as in $S^{\prime}$. Therefore, $H^{\prime}$ satisfies $\Gamma$-opacity if and only if $H^{\prime}$ is view-serializable.

\subsection{Online Opacity-Permissiveness}

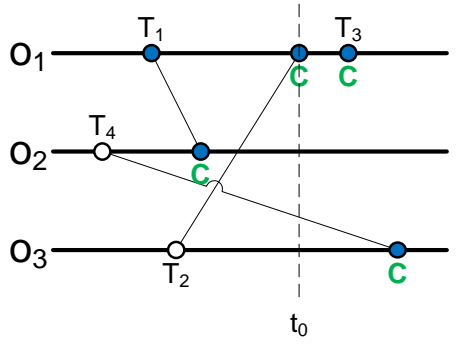

Figure 3: The order of transactions $T_{1}$ and $T_{2}$ is changed after their commit time.

Intuitively, the problem with strict online opacity-permissiveness lies in the fact that the order of committed transactions may be undefined and may change in the future. Consider, for example, the scenario depicted in Figure 3. Transactions $T_{1}$ and $T_{2}$ are not ordered according to real-time order, therefore they are not ordered by $\Gamma$. At time $t_{0}$, the serialization order is $T_{1} \rightarrow T_{2}$, as $o_{1}$ holds the value written by $T_{2}$. When $T_{3}$ commits, the serialization order of $T_{1}$ and $T_{2}$ becomes undefined, since $T_{3}$ overwrites $o_{1}$ before any transaction reads the value written by $T_{2}$. And when $T_{4}$ commits, the serialization order becomes $T_{2} \rightarrow T_{4} \rightarrow T_{1} \rightarrow T_{3}$. If the partial serialization order induced by the run cannot change after being defined, the problem becomes much easier. We capture this intuition with the following definition:

Definition 2. A TM satisfies $\lambda$-persistent ordering for a partial order $\lambda$ iffor every run $\lambda$ is updated according to the following rules: (1) at the beginning of the run $\lambda$ is empty; (2) at any point in time $\lambda$ is a transitive 
closure over a relation ordering exactly all the pairs of $T_{i}, T_{j}$, s.t. write $\left(T_{i}\right) \cap \operatorname{write}\left(T_{j}\right) \neq \emptyset$; and (3) each time $\lambda$ is updated, its new value preserves the one previously defined.

In other words, if $T_{i}$ and $T_{j}$ are committed transactions in $H$ that have written to the same object in a given TM, then they are ordered by $\lambda$ and their order will persist in every extension of the run.

We now define a more relaxed property, online $\Gamma$-opacity-permissiveness, which may be satisfied at a reasonable implementation cost.

Definition 3. A TM satisfies online $\Gamma$-opacity-permissiveness for a given $\Gamma$ if the TM satisfies $\lambda$-persistent ordering for some $\lambda$ consistent with $\Gamma$, and the TM forcefully aborts a set $S$ of live transactions only when aborting any subset $S^{\prime} \subset S$ of transactions violates $(\Gamma \cup \lambda)$-opacity.

Note that Definition 3 implies that each committing transactions should define its serialization order with regard to all other committed transactions that have written to the same objects. To the best of our knowing, all existing TMs do in fact define the order on two transactions that write to the object by the time the latter commits. We note that this requirement might be limiting for TMs that wish to exploit the benefits of commutative or write-only operations (see [11]), and do not necessarily define the serialization point of the committed transactions. However, this limitation is essential for an effective check of the opacity criterion.

In the following sections we show a polynomial-time TM satisfying online opacity-permissiveness. We now prove that such an implementation, nevertheless, has some inherent costs.

One of the basic decisions that needs to be made during the design of a TM is whether to expose the fact that transaction $T_{i}$ has read the object $o$, i.e. make a change in shared memory as a result of the read, making the read visible. In case we expose the read, there arises another question, regarding whether we can postpone exposing the read until the commit. One of the central problems with exposing the read is that it requires writing metadata in shared memory. One typically tries to avoid writes to shared memory, because writing data that is read by different cores has a high cache penalty. Postponing exposing the read until the commit may save redundant writes in case the transaction eventually aborts.

Unfortunately, if a TM satisfying online opacity-permissiveness does not expose a read operation immediately when the read happens, then all read operations through the entire execution must return the respective objects' initial values, rendering such a TM implementation completely useless:

Lemma 4. For any $\Gamma$, if a TM satisfies online $\Gamma$-opacity-permissiveness and never exposes read operations when the reads happen, then no transaction $T_{i}$ can read from a committed transaction $T_{j}$ if $T_{j}$ has written to more than one object.

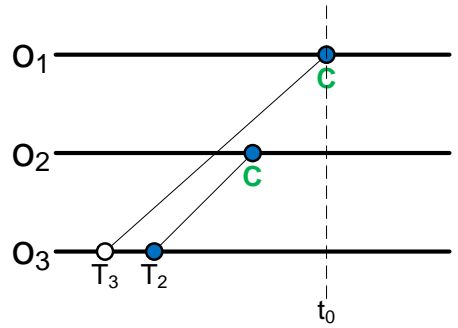

(a) Run $r_{1}: T_{3}$ commits,

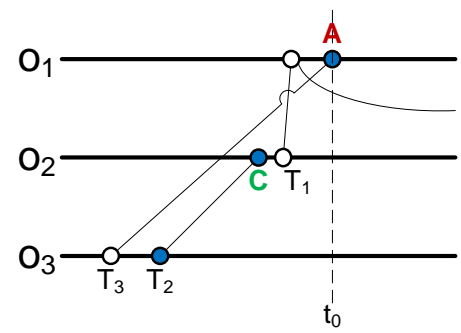

(b) Run $r_{2}: T_{3}$ cannot commit

Figure 4: $T_{3}$ does not distinguish between $r_{1}$ and $r_{2}$ at time $t_{0}$. If $T_{1}$ does not expose its reads, it is not allowed to read the value written by $T_{2}$, because otherwise the commit of $T_{3}$ would violate $\Gamma$-opacity. 
Proof. Assume by contradiction that there exists a TM, satisfying online $\Gamma$-opacity-permissiveness that does not expose read operations when the reads happen, and which allows transactions to read values written by the transactions with more than one object in the write-set. Consider run $r_{1}$ depicted in Figure 4. Transaction $T_{3}$ reads $o_{3}$ without exposing it. Afterwards transaction $T_{2}$ writes to $o_{3}$ and $o_{2}$. Then $T_{3}$ writes to $o_{1}$ and tries to commit. We next construct $r_{2}$, where transaction $T_{1}$ reads the value written by transaction $T_{2}$, as depicted in Figure 4(b). By our assumption, this value can indeed be read by some transaction. $T_{1}$ then continues to read $o_{1}$. Note that $T_{1}$ is not aware of transaction $T_{3}$ preceding $T_{2}$ because $T_{3}$ did not expose its read. All the transactions start at the same time and therefore cannot be ordered by real-time order. In run $r_{1}, T_{3}$ must commit because the run satisfies $\Gamma$-opacity. In run $r_{2}$, however, $T_{3}$ cannot commit because that would create a precedence cycle $T_{1} \rightarrow T_{3} \rightarrow T_{2} \rightarrow T_{1}$ and thus violate $\Gamma$-opacity. But since we assumed that reads are invisible, runs $r_{1}$ and $r_{2}$ are indistinguishable to $T_{3}$ at time $t_{0}$, a contradiction.

\section{The AbortsAvoider Algorithm}

We now present AbortsAvoider, a TM algorithm implementing online opacity-permissiveness. The basic idea behind AbortsAvoider is to maintain a precedence graph of transactions, and keep it acyclic, as explained in Section 6.1. A simplified version of the protocol based on this graph is then presented in Section 6.2. The key challenge AbortsAvoider faces is that completed transactions cannot always be removed from the graph, whereas keeping all transactions forever is clearly impractical. We address this challenge in Section 6.3, presenting a garbage collection mechanism for removing terminated transactions from the graph. In Section 6.4 we present another optimization, which shortens paths in the graph to reduce the number of terminated transactions traversed during the acyclity check. Our complexity analysis appears in the same section.

\subsection{Basic Concept: Precedence Graph}

Information bookkeeping. Our protocol maintains object version lists. We now explain what such a TM does: (1) each object $o$ is associated with a totally ordered set of versions, (2) a read of $o$ returns the value of one of $o$ 's versions, and (3) a write to $o$ adds a new version of $o$ upon commit. For simplicity, at any given moment, we number the versions of the object in increasing order. (Note that the numbering is for analysis purposes only, and the numbers of the versions change during the run as the versions are inserted and removed from the versions list). The object version $o . v_{n}$ includes the data, o. $v_{n}$.data, the writer transaction, o. $v_{n}$.writer, and a set of readers, o. $v_{n}$.readers. Each transaction has a readList and a writeList. An entry in a readList points to the version that has been read by the transaction. A writeList entry points to the object that should be updated after commit, the new data, and the place to insert the new version, (which may be undefined till the commit).

Precedence graph. Transactions may point to one another, forming a directed labelled precedence graph, $P G$. $P G$ reflects the dependencies among transactions as created during the run. The vertexes of $P G$ are transactions, the edges of $P G$ are as follows (Figure 5):

If $\left(T_{j}, T_{i}\right) \in \Gamma$, then $P G$ contains $\left(T_{j}, T_{i}\right)$ labelled $L_{\Gamma}$ ( $\Gamma$ order). If $T_{i}$ reads o. $v_{n}$ and $T_{j}$ writes o. $v_{n}$, then $P G$ contains $\left(T_{j}, T_{i}\right)$ labelled $L_{R a W}$ (Read after Write). If transaction $T_{i}$ writes $o . v_{n}$ and $T_{j}$ writes $o . v_{n-1}$, then $P G$ contains $\left(T_{j}, T_{i}\right)$ (Write after Write) labelled $L_{W a W}$. If transaction $T_{i}$ writes $o . v_{n}$ and $T_{j}$ reads $0 . v_{n-1}$, then $P G$ contains $\left(T_{j}, T_{i}\right)$ labelled $L_{W a R}$ (Write after Read). 


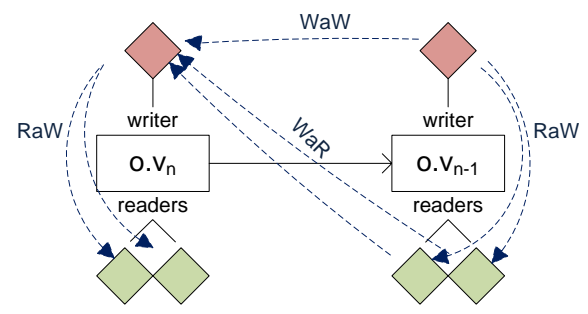

Figure 5: Object versions and the precedence graph, $P G$.

Below we present lemmas that link maintaining acyclity in $P G$ and satisfying online-permissiveness. To this end, we restrict our discussion to non-local histories, which we now define. We say that a read operation of $T_{i} \operatorname{read}_{i}(o)$ in $H$ is local if it is preceded in $H \mid T_{i}$ by a write operation write $_{i}(o, v)$. A write operation write $_{i}(o, v)$ is local if it is followed in $H \mid T_{i}$ by another write operation write $_{i}(o, v$ '). The non-local history of $H$ is the longest subsequence of $H$ not containing local operations [8]. Note that the precedence graph does not refer to local operations.

Lemma 5. Consider a TM maintaining object version lists. If $P G$ is acyclic throughout some run, then the non-local history $H$ of the run satisfies $\Gamma$-opacity.

Proof. Let $H$ be a history over transactions $\left\{T_{1} \ldots T_{n}\right\}$. Let $H_{C}=\operatorname{Complete}(H)$, i.e. $H$ with $A_{i}$ added for every live $T_{i} \in H$.

Since $P G$ is acyclic, it can be topologically sorted. Let $T_{i 1}, \ldots, T_{i n}$ be a topological sort of $P G$, and let $S$ be the sequential history $T_{i 1}, \ldots, T_{i n}$. Clearly, $S$ is equivalent to $H_{C}$ because both of the histories contain the same transactions and each transaction issues the same operations and receives the same responses in both of them.

We now prove that every $T_{i} \in S$ is legal. Assume by contradiction that there are non-legal transactions in $S$. Let $T_{i}$ be the first such transaction. If $T_{i}$ is non-legal, $T_{i}$ reads a value of object $o$ that is not the latest value written to $o$ in $S$ by a committed transaction. (Recall that by definition of object version lists, only values written by committed transactions can be read.) $S$ contains only non-local operations, and therefore $T_{i}$ reads the version $0 . v_{n}$ written by another transaction $T_{j}$. Therefore, there is an edge from $T_{j}$ to $T_{i}$ in $P G$. It follows that $T_{j}$ is committed in $S$ and ordered before $T_{i}$ according to the topological sort. If the value of $o . v_{n}$ is not the latest value written in $S$ before $T_{i}$, then there exists another committed transaction $T_{j}^{\prime}$ that writes to $o$ and is ordered between $T_{j}$ and $T_{i}$ in $S$. If $T_{j}^{\prime}$ writes to a version earlier than $o . v_{n}$, then there is a path from $T_{j}^{\prime}$ to $T_{j}$ in $P G$, and therefore $T_{j}^{\prime}$ is ordered before $T_{j}$ in $S$. If $T_{j}^{\prime}$ writes to a version later than $o . v_{n}$, then there is a path from $T_{i}$ to $T_{j}^{\prime}$ in $P G$, and therefore $T_{j}^{\prime}$ is ordered after $T_{i}$ in $S$. In any case, $T_{j}^{\prime}$ cannot be ordered between $T_{j}$ and $T_{i}$ in $S$, a contradiction.

Finally, for each pair $T_{i} \prec_{\Gamma} T_{j}, P G$ contains an edge from $T_{i}$ to $T_{j}$. Therefore, according to the topological sort, $S$ preserves the partial order $\Gamma$.

Summing up, Complete $(\mathrm{H})$ is equivalent to a legal sequential history $S$, and $S$ preserves partial order $\Gamma$. Therefore $H$ is $\Gamma$-opaque.

We define $\lambda_{P G}$ to be the following binary relation: if $P G$ contains a path from $T_{i}$ to $T_{j}$ consisting of $L_{W a W}$ edges, then $T_{i} \prec_{\lambda_{P G}} T_{j}$. Note that if $P G$ is acyclic, then $\lambda_{P G}$ is reflexive, antisymmetric and transitive, and therefore $\lambda_{P G}$ is a partial order.

Lemma 6. Every TM that maintains object version lists and keeps $P G$ acyclic satisfies $\lambda_{P G \text {-persistent }}$ ordering. 
Proof. We will show that every TM maintaining object version lists and keeping $P G$ acyclic satisfies the three necessary conditions for $\lambda_{P G}$-persistent ordering.

(1) Initially $P G$ does not contain edges and thus $\lambda_{P G}$ is empty.

(2) Consider an arbitrary point during the run. Let $\chi$ be the transitive closure of the partial order containing exactly all the pairs of transactions $T_{i}, T_{j}$ committed up to this point, s.t. write $\left(T_{i}\right) \cap$ write $\left(T_{j}\right) \neq \emptyset$, where $T_{i} \prec_{\chi} T_{j}$ iff $T_{j}$ writes a higher numbered version than $T_{i}$. We show that $\lambda_{P G}$ is equal to $\chi$.

We show first that $\lambda_{P G} \supseteq \chi$. Consider two committed transactions $T_{i} \prec_{\chi} T_{j}$ that have a common object $o$ in their write-sets, s.t. $T_{i}$ has written to the version $o . v_{i}$ and $T_{j}$ has written to the version $o . v_{j}$, where $i<j$. Then $P G$ contains a path from $T_{i}$ to $T_{j}$ consisting of $L_{W a W}$ edges and therefore $\lambda_{P G}$ contains a pair $\left(T_{i}, T_{j}\right) . \lambda_{P G}$ is transitive by definition, and therefore $\lambda_{P G} \supseteq \chi$. It remains to show that $\lambda_{P G} \subseteq \chi$. If $T_{i} \prec \lambda_{P G} T_{j}$, then $P G$ contains a path from $T_{i}$ to $T_{j}$ consisting of $L_{W a W}$ edges. Every edge on the path defines a pair of committed transactions that have written to the same object and therefore are ordered by $\chi$. By transitivity of $\chi$, we conclude that $T_{i} \prec_{\chi} T_{j}$. We have shown that both $\lambda_{P G} \supseteq \chi$ and $\lambda_{P G} \subseteq \chi$. Therefore, $\lambda_{P G}=\chi$.

(3) Finally, edges are never removed from $P G$, therefore each time $\lambda_{P G}$ is updated, its new value preserves the previously defined order.

Lemma 7. Consider a TM maintaining object version lists that forcefully aborts a set $S$ of live transactions only when aborting any subset $S^{\prime} \subset S$ of transactions creates a cycle in $P G$. Then this TM satisfies online Г-opacity-permissiveness.

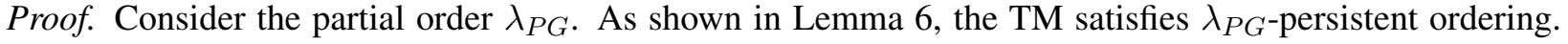
We need to show that if there is a cycle in $P G$, then the run violates $\left(\Gamma \cup \lambda_{P G}\right)$-opacity.

We show first that if there is an edge $\left(T_{i}, T_{j}\right)$ in $P G$, then every legal sequential history $S$ preserving $\Gamma \cup \lambda_{P G}$ and equivalent to Complete $(H)$ orders $T_{i}$ before $T_{j}$. Consider two transactions $T_{i}$ and $T_{j}$ s.t. there is an edge $\left(T_{i}, T_{j}\right)$ in $P G$. If the edge is labelled $L_{\Gamma}$, then $\left(T_{i}, T_{j}\right) \in \Gamma$, and $S$ orders $T_{i}$ before $T_{j}$. If the edge is labelled $L_{R a W}$, then $T_{j}$ reads a value written by $T_{i}$ and $S$ also orders $T_{i}$ before $T_{j}$. If the edge is labelled $L_{W a W}$, then $T_{i}<T_{j}$ according to $\lambda_{P G}$, hence $S$ also orders $T_{i}$ before $T_{j}$. If the edge is labelled $L_{W a R}$, then $T_{i}$ reads $o . v_{n}$ while $T_{j}$ writes $o . v_{n+1}$. On the one hand, $T_{j}$ should be ordered after $o . v_{n}$. writer in $S$ (there is an edge from $o . v_{n}$.writer to $T_{j}$ labelled $L_{W a W}$ ). On the other hand, $T_{j}$ cannot be ordered between $o . v_{n}$.writer and $T_{i}$, because $T_{i}$ must read the value written by o. $v_{n}$.writer in $S$. Therefore, $T_{j}$ is ordered after $T_{i}$ in $S$ in this case as well.

Summing up, an edge $\left(T_{i}, T_{j}\right)$ in the precedence graph induces the order of $T_{i}$ before $T_{j}$ in any legal sequential history $S$ preserving $\Gamma \cup \lambda_{P G}$ and equivalent to Complete $(H)$. Therefore, if $P G$ contains a cycle, no such sequential history exists, and the TM cannot satisfy $\Gamma \cup \lambda$-opacity.

Corollary 1. Consider a TM maintaining object version lists that keeps PG acyclic and forcefully aborts a set $S$ of live transactions only when aborting any subset $S^{\prime} \subset S$ of transactions creates a cycle in $P G$. Then this TM satisfies $\Gamma$-opacity and online $\Gamma$-opacity-permissiveness.

\subsection{Simplified $\Gamma$-AbortsAvoider Algorithm}

AbortsAvoider algorithm maintains object version lists as explained above, keeps $P G$ acyclic and forcefully aborts a transaction only if not aborting any transaction would create a cycle in $P G$. Read and write operations are straightforward, they are depicted in Algorithm 1. A read operation (line 4) looks for the latest possible object version to read without creating a cycle in $P G$. Write operations (line 13) postpone the actual work till the commit. 


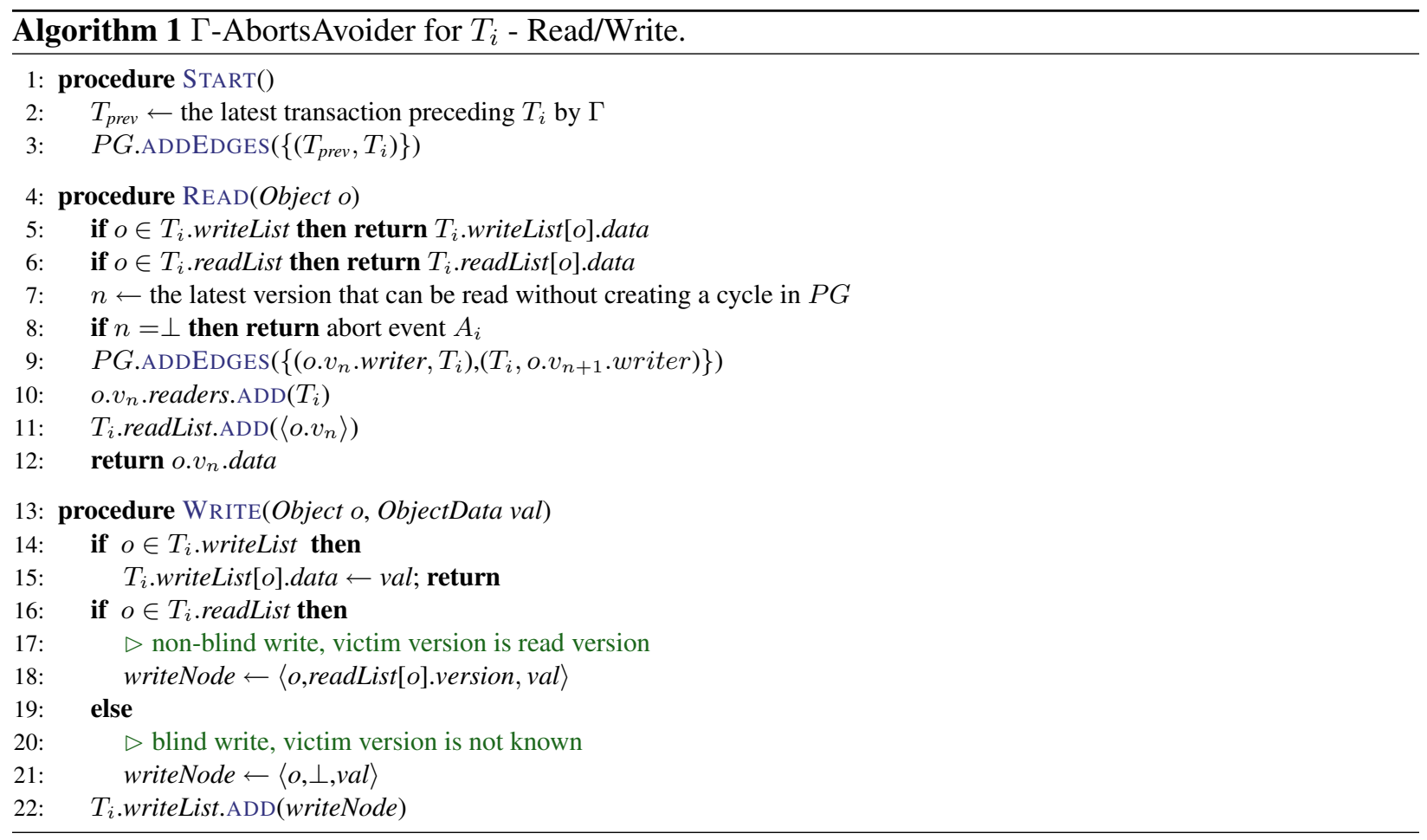

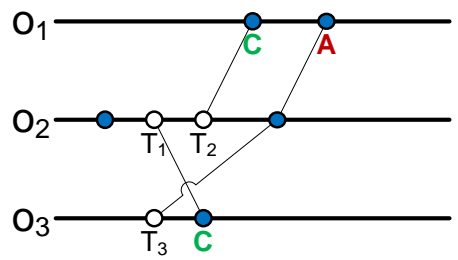

(a) Run with greedy check

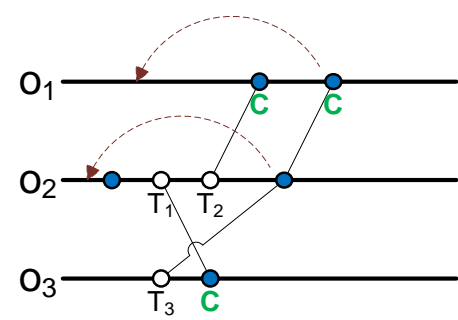

(b) Run with no spare aborts

Figure 6: Checking the written objects in a greedy way during the commit may lead to a spare abort.

The commit operation is more complicated. Intuitively, for each object written during transaction, the algorithm should find a place in the object's version list to insert the new version without creating a cycle. Unfortunately, checking the objects one after another in a greedy way can lead to spare aborts, as we illustrate in Figure 6(a). Committing $T_{3}$ first seeks for a place to install the new version of $o_{1}$ and decides to install it after the last one (serializing $T_{3}$ after $T_{2}$ ). When $T_{3}$ considers $o_{2}$, it discovers that the new version cannot be installed after the last one, because $T_{3}$ should precede $T_{1}$, but it also cannot be installed before the last one, because that would make $T_{3}$ precede $T_{2}$, so $T_{3}$ is aborted. However, installing the new version of $o_{1}$ before the last one would have allowed $T_{3}$ to commit, as depicted in Figure 6(b), that is why aborting $T_{3}$ violates online $\Gamma$-opacity-permissiveness.

Our commit operation (Algorithm 2, line 23) is divided to two phases. We call the object version after which the new version is to be installed a victim version. The victim version is known only for the non-blind writes (that is version, which has been read before the write, line 18). In the first phase the algorithm tries to install the non-blind writes (lines 27-33). In the second phase (lines 35-48) the algorithm tries to find the 
vicim versions for the blind writes in iterations. Initially, the victim is the object's latest version. In each iteration, the algorithm traverses the objects and for each one searches for the latest possible victim to install the new version without creating a cycle in $P G$ (line 40). When victim $o . v_{n}$ is found, an edge from $T_{i}$ to the writer of $o . v_{n+1}$ is added to $P G$ (line 46). We add only the outgoing edges at this point, because changing the victim from $o . v_{n}$ to $o . v_{n-1}$ may remove some incoming edges to $T_{i}$ but cannot remove outgoing ones. Meanwhile, incoming edges are kept in inEdges. After each iteration, there are possibly new outgoing edges added to $P G$, that would mean that the previously found victim versions might not suit anymore and a new iteration should be run. Once there is an iteration when no new edges are added, the algorithm commits - it installs the new versions after their victims and adds all the edges, including inEdges from the latest iteration, to the $P G$.

The following lemma immediately follows from the protocol.

Lemma 8. $\Gamma$-AbortsAvoider maintains $P G$ acyclic.

Proof. The edges added to the graph are defined in functions READ (line 7) and VALIDATEWRITE (line 63). Both functions validate that adding the new edges preserves $P G$ acyclity.

We now want to show that the algorithm does not introduce unnecessary abotrs.

Theorem 2. $\Gamma$-AbortsAvoider forcefully aborts a transaction only if not aborting any transaction would create a cycle in $P G$.

Proof. The read operation of object $o$ (line 4) returns $A_{i}$ only if there is no object version to read without introducing a cycle in $P G$. Write operation (line 13) does not abort any transaction - it postpones all the work till the commit.

Commit operation (line 23) tries to write the new versions of all the objects written during the transaction. If the object is written in the non-blind way, then the victim version is known beforehand and the new version has to be installed after the version that has been read (line 29). In this case the validation is done by validateWrite function (line 63), which fails if and only if adding the appropriate edges to $P G$ creates a cycle.

It remains to show that commit function does not succeed to execute the blind writes only if that creates a cycle in $P G$. We will show now that if there exists a way to execute the blind writes without creating a cycle in $P G$, the algorithm will find it.

First of all, we will analyze the variable newEdges (line 24), which keeps the set of the edges added to $P G$ upon successful commit. Edge $\left(T_{i}, T_{j}\right) \in$ newEdges is compulsory, if $P G$ must have a path from $T_{i}$ to $T_{j}$ after successful commit (to that end, the edge represents a real, compulsory dependency).

Lemma 9. During COMMIT() function of AbortsAvoider algorithm, newEdges set contains compulsory edges only.

Proof. In the first phase of Commit(), AbortsAvoider proceeds the non-blind writes (lines 27-33). There is a single possible victim version for the non-blind write, and therefore the edges added to newEdges set during the first phase are compulsory.

Consider the second phase of COMmiт(), when AbortsAvoider proceeds the blind writes (lines 35-48). We will show by induction that all the edges added to newEdges in the second phase are compulsory.

Induction basis. At the beginning of the second phase newEdges set contains only the edges added by the non-blind writes, which are compulsory, as shown before.

Induction step. Let's assume, that all the edges added to newEdges by the algorithm so far are compulsory. Consider new edge $\left(T_{i}, o . v_{k+1}\right.$.writer $)$ added to newEdges by the algorithm in line 46 . This happens 
if $o . v_{k}$ is chosen to be a victim version for writing to object $o$. According to the algorithm, o. $v_{k}$ is chosen to be the victim version only if all the versions $o . v_{k^{\prime}}$ for $k^{\prime}>k$ did not suit to be the victim versions for a given newEdges set. According to the induction assumption, newEdges set contains compulsory edges only, therefore all the versions $0 . v_{k^{\prime}}$ for $k^{\prime}>k$ cannot be victim versions for the write operation. According to the algorithm, choosing any object version $o . v_{k^{\prime}}$ for $k^{\prime} \leq k$ (i.e., object version that is earlier than $o . v_{k}$ ) yields a path from $T_{i}$ to $o . v_{k+1}$.writer in $P G$, finishing the proof.

For each object written in a blind way the algorithm checks the victim versions starting from the latest one. Victim version validation is executed in the following way: $P G$ is checked for acyclity after inserting the edges from newEdges set together with the edges corresponding to adding the new version after o. $v_{k}$. As stated in Lemma 9, newEdges set contains compulsory edges only, therefore validation fail for $o . v_{k}$ means that neither $o . v_{k}$, nor any version later than $o . v_{k}$ can be the victim version of $o$. The algorithm traverses the objects in iterations, till it finds a combination of victim versions that does not create a cycle in $P G$ (and then commits), or discovers object $o$ s.t. none of $o$ 's versions can be the victim version (and then aborts).

Corollary 2. $\Gamma$-AbortsAvoider satisfies $\Gamma$-opacity and online $\Gamma$-opacity-permissiveness.

We have shown that $\Gamma$-AbortsAvoider protocol is correct and avoids unnecessary aborts. In the rest of the paper we will show the garbage collection rules and optimization techniques for the protocol.

\subsection{Garbage Collection}

A TM should garbage collect unused metadata. In this section, we describe how terminated transactions may be garbage collected.

Read operations. Consider transaction $T_{i}$ reading object $o$. The following lemma stipulates that some of the edges added to the precedence graph in the simplified protocol are redundant, and in fact, the only edges that need to be added by the protocol during read operations are incoming ones.

Lemma 10. When $T_{i}$ reads o. $v_{n}$, it suffices to add one edge from o. $v_{n}$. writer to $T_{i}$ in $P G$.

Proof. We say that adding an edge $\left(v_{1}, v_{2}\right)$ is unnecessary, if $P G$ already contains a path from $v_{1}$ to $v_{2}$, thus adding this edge does not influence on the cycle detection. We will show that adding the outgoing edge from $T_{i}$ to $o . v_{n}$.writer during a read is unnecessary. Therefore the only edge that need to be added by the protocol is the edge from $o . v_{n-1}$.writer to $T_{i}$.

The protocol adds outgoing edge from $T_{i}$ to $o . v_{n}$. writer if $T_{i}$ reads version $o . v_{n-1}$. According to the algorithm, $T_{i}$ tries first to read the latest version $o . v_{n+k}$, if this read creates a cycle, it tries to read $o . v_{n+k-1}$, $o . v_{n+k-2}$ and so on till it arrives to $o . v_{n-1}$. Note, that before starting the read, the graph $P G$ was acyclic. If $T_{i}$ does not succeed to read $o . v_{n+k}$, it means that adding an edge from $o . v_{n+k}$.writer to $T_{i}$ would create a cycle, hence there is a path from $T_{i}$ to $o . v_{n+k}$.writer before the start of the read. When $T_{i}$ tries to read $o . v_{n+k-1}$ and does not succeed, it means that adding the edges $\left\{\left(o . v_{n+k-1}\right.\right.$. writer, $\left.T_{i}\right),\left(T_{i}, o . v_{n+k}\right.$. writer $\left.)\right\}$ creates a cycle in $P G$. As we have concluded, before the read, $P G$ contained a path from $T_{i}$ to $o . v_{n+k}$.writer and was acyclic, therefore adding the single edge $\left(o . v_{n+k-1}\right.$. writer, $\left.T_{i}\right)$ creates a cycle in $P G$, i.e. there was a path from $T_{i}$ to $o . v_{n+k-1}$.writer before the read. Continuing in the same way, we conclude that before the read there was a path from $T_{i}$ to o. $v_{n}$.writer. Therefore, adding an edge from $T_{i}$ to o. $v_{n}$. writer is unnecessary.

Using the optimization above, no incoming edge is ever added to a terminated transaction as a result of a read operation. 
Write operations. We would like to know whether the new incoming edges may be added to a terminated transaction as a result of write operation. Consider committed transaction $T_{i}$ that has written to $o$. If the new version $o . v_{n}$ has been written in a non-blind way (i.e. transaction $T_{i}$ has read the version $o . v_{n-1}$ and then installed $o . v_{n}$ ), then no other transaction $T_{j}$ will be able to install a new version between $o . v_{n-1}$ and $o . v_{n}$, for that would cause a cycle between $T_{i}$ and $T_{j}$. Blind writes, however, are more problematic. Consider, for example, the scenario depicted in Figure 7. At time $t_{0}, T_{1}$ has no incoming edges, but we are still not allowed to garbage collect it as we now explain. There is a transaction $T_{2}$ that read object $o_{1}$ with a live preceding transaction $T_{3}$. At the time of $T_{3}$ 's commit, it discovers that it cannot install the last version of $o_{1}$, and tries to install the preceding version. Had we removed $T_{1}$ from $P G$, this would have caused a consistency violation, because we would miss the cycle between $T_{1}$ and $T_{3}$.

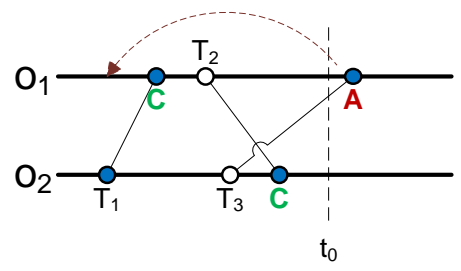

Figure 7: The blind write of transaction $T_{1}$ does not allow us to garbage collect it at time $t_{0}$.

The example above demonstrates the importance of knowing that from some point onward, $T_{i}$ may have no new incoming edges. The lemma below shows that some edge additions can be saved:

Lemma 11. If $T_{i}$ is a terminated transaction, then no incoming edges need to be added to $T_{i}$ in $P G$ as long as for each o. $v_{n}$ written blindly by $T_{i}$ there is no reader with a live preceding transaction.

Proof. Consider a terminated transaction $T_{i}$ satisfying conditions of the lemma. According to Lemma 10 no transaction may add incoming edges to $T_{i}$ as a result of read operation. It remains to check the writes. According to the protocol, the incoming edge to $T_{i}$ may be added only if transaction $T_{j}$ installs the version prior to the version $o . v_{n}$ written by $T_{i}$. First of all we should notice that $o . v_{n}$ should be written in a blind way in order to make this scenario happen. Secondly, if $T_{j}$ tries to insert a new version before $0 . v_{n}$, it means that $T_{j}$ failed to insert its version after $o . v_{n}$, i.e. adding the edges from $T_{i}$ and from the readers of $o . v_{n}$ to $T_{j}$ created a cycle. But we know that $T_{j}$ cannot precede the readers of $o . v_{n}$ according to the condition of the lemma, that is why there was a path from $T_{j}$ to $T_{i}$ before the write operation of $T_{j}$. Therefore there is no need to add the edge from $T_{j}$ to $T_{i}$ when installing the new version.

Garbage collection conditions. We say that a transaction is stabilized if no incoming edges may be added to it in the future. At the moment when $T_{i}$ has no incoming edges and it is stabilized, we know that $T_{i}$ will not participate in any cycle, and thus may be garbage collected.

Theorem 3. The terminated transaction $T_{i}$ is stabilized at time $t_{0}$ if either (1) $T_{i}$ has not written blindly any object version $0 . v_{n}$, or (2) all live transactions at time $t_{0}$ and all the transactions beginning after $t_{0}$ follow $T_{i}$ according to $\Gamma$.

Proof. According to Lemma 11, no incoming edges need to be added to terminated $T_{i}$ in $P G$ if $T_{i}$ has no blind writes. If transaction $T_{j}$ follows $T_{i}$ according to $\Gamma$, then according to AbortsAvoider algorithm, $P G$ will contain a path from $T_{i}$ to $T_{j}$ after $\mathrm{START}\left(\right.$ ) operation of $T_{j}$. Therefore, $T_{j}$ may not add incoming edge 
to $T_{i}$ if $T_{i} \prec_{\Gamma} T_{j}$. Hence, if all live transactions at $t_{0}$ and all the transactions beginning after $t_{0}$ follow $T_{i}$ according to $\Gamma$, then no new incoming edges will be added to $T_{i}$.

For this, we deduce that terminated transactions with no incoming edges satisfying one of the conditions of Theorem 3 may be garbage collected. Note that in the runs with no blind writes, every terminated transaction is stabilized and thus the transaction may be garbage collected at the moment it has no incoming edges.

\subsection{Path Shortening and Runtime Analysis}

AbortsAvoider protocol allows adding new edges to $P G$ only if they do not introduce cycles in $P G$. The straightforward cycle detection algorithm runs DFS starting from $T_{i}$, traversing a set of nodes we refer to as ingress $_{i}$. We now present an optimization that reduces the number of nodes in ingress . $_{\text {. }}$

Consider stabilized terminated $T_{j}$. The idea is to connect the ingress nodes to the egress nodes of $T_{j}$ directly, thus preventing DFS from traversing $T_{j}$. This becomes possible because $T_{j}$ is stabilized and thus may not have new ingress nodes, hence the egress nodes do not miss the precedence info when they lose their edges from $T_{j}$. Once a terminated transaction $T_{j}$ satisfies the conditions of Lemma 11 and it can no longer have additional incoming edges, (e.g., any transaction with no blind writes), we remove all of its outgoing edges by connecting its ingress nodes directly to its egress nodes as described above, and indicate that $T_{j}$ is a sink, i.e., cannot have outgoing edges in the future. Once a transaction is marked as a sink, any outgoing edge that should be added from it is instead added from its ingress nodes. Note that our path shortening only bypasses stabilized nodes. Had we bypassed also non-stabilized ones, we would have had to later deal with adding new incoming nodes to their egress nodes, which could require a quadratic number of operations in the number of terminated transactions. Hence, we chose not to do that.

Runtime complexity of the operations. Running DFS on ingress $s_{i}$ takes $O\left(V^{2}\right)$, where $V$ is the number of transactions preceding $T_{i}$, whose nodes have not been garbage collected. In the general case, $V=$ \#terminated + \#live. But if all the transactions preceding $D s c_{i}$ had no blind writes, $V=$ \#live.

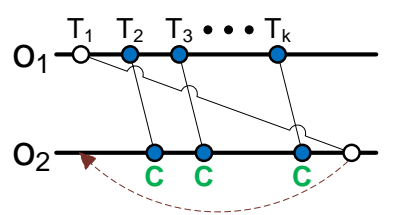

Figure 8: All object versions must be kept, as their writers have a live preceding transaction $T_{2}$.

The read operation seeks the proper version to read in the version list. Unfortunately, the number of versions that need to be kept is limited only by the number of terminated transactions. Consider the scenario depicted in Figure 8. Here, the only version of $o_{2}$ that may be read by $T_{1}$ is the first, all other versions are written by transactions that $T_{1}$ precedes. In order to find a latest suitable version, the read operation may use a binary search $-O(\log ($ \#terminated $))$ versions should be checked. Adding the edges takes $O$ (\#live). So altogether, the read complexity is $O\left(\log (\# t e r m i n a t e d) \cdot \max \left\{\# l i v e^{2}, \#_{\text {terminated }}^{2}\right\}\right)$, and $O\left(\log (\# t e r m i n a t e d) \cdot \# l i v e^{2}\right)$ when there are no blind writes.

The write operation postpones all the work till the commit. The number of iterations in the commit phase is $O$ (\#writes · \#terminated $)$, and in each iteration $O$ (\#writes) validate operations should be run. So 
the overall write cost is $O\left(\#\right.$ writes $^{2} \cdot \#$ terminated $\left.\cdot \max \left\{\# l i v e^{2}, \# t e r m i n a t e d^{2}\right\}\right)$, and $O\left(\# l i v e^{2}\right)$ when there are no blind writes.

Finally, we would like to emphasize that although in the worst-case, these costs may seem high, transactions without blind writes are garbage collected immediately upon commit. Moreover, the only nodes in ingress $_{i}$ where cycles are checked are transactions that conflict with $T_{i}$. Typically, in practice, the number of such conflicts is low, suggesting that our algorithm's common-case complexity is expected to be good. On the other hand, if the number of conflicts is high, then most TMs existing today would abort one of the transactions in each of these cases, which is not necessarily a better alternative.

\section{Conclusions}

The paper took a step towards providing a theory for understanding TM aborts, by investigating what kinds of spare aborts can or cannot be eliminated, and what kinds can or cannot be avoided efficiently. We have shown that some unnecessary aborts cannot be avoided, and that there is an inherent tradeoff between the overhead of a TM and the extent to which it reduces the number of spare aborts: while strict online opacitypermissiveness is NP-hard, we presented a polynomial time algorithm AbortsAvoider, satisfying the weaker online opacity-permissiveness property. Understanding the properties of spare aborts is still far from being complete. For example, relaxations of the online opacity-permissiveness property or restrictions of the workload may be amenable to more efficient solutions. Moreover, the implications of the inherent "spare aborts versus time complexity" tradeoff we have shown are yet to be studied.

\section{Acknowledgments}

We would like to thank Hagit Attiya, Eshcar Hilel and Alessia Minali, as well as the anonymous reviewers for their helpful comments.

\section{References}

[1] H. Attiya, L. Epstein, H. Shachnai, and T. Tamir. Transactional contention management as a non-clairvoyant scheduling problem. In PODC '06: Proceedings of the twenty-fifth annual ACM symposium on Principles of distributed computing, pages 308-315, New York, NY, USA, 2006. ACM.

[2] U. Aydonat and T. Abdelrahman. Serializability of transactions in software transactional memory. In Second ACM SIGPLAN Workshop on Transactional Computing, 2008.

[3] D. Dice, O. Shalev, and N. Shavit. Transactional locking 2. In Proceedings of the 20th International Symposium on Distributed Computing, 2006.

[4] R. Ennals. Cache sensitive software transactional memory. Technical report, Intel.

[5] K. Fraser. Practical lock-freedom. Technical report, Cambridge, 2004.

[6] V. Gramoli, D. Harmanci, and P. Felber. Toward a theory of input acceptance for transactional memories. In Proceedings of the 12th International Conference On Principles Of Distributed Systems (OPODIS'08), volume 5401 of LNCS, pages 527-533. Springer-Verlag, dec 2008.

[7] R. Guerraoui, T. A. Henzinger, and V. Singh. Permissiveness in Transactional Memories. In DISC $2008,2008$.

[8] R. Guerraoui and M. Kapalka. On the correctness of transactional memory. In Proceedings of the 13th ACM SIGPLAN Symposium on Principles and practice of parallel programming, pages 175-184, 2008. 
[9] M. Herlihy, V. Luchangco, M. Moir, and W. N. Scherer, III. Software transactional memory for dynamic-sized data structures. In PODC '03: Proceedings of the twenty-second annual symposium on Principles of distributed computing, pages 92-101, New York, NY, USA, 2003. ACM.

[10] M. Herlihy and J. E. B. Moss. Transactional memory: architectural support for lock-free data structures. SIGARCH Comput. Archit. News, 21(2):289-300, 1993.

[11] J. E. B. Moss. Open nested transactions: Semantics and support. In Workshop on Memory Performance Issues (WMPI'06), 2006.

[12] J. Napper and L. Alvisi. Lock-free serializable transactions. Technical report, The University of Texas at Austin, 2005.

[13] C. H. Papadimitriou. The serializability of concurrent database updates. J. ACM, 1979.

[14] T. Riegel, C. Fetzer, H. Sturzrehm, and P. Felber. From causal to z-linearizable transactional memory. Technical report, Université de Neuchâtel, Institut d'Informatique, 2007.

[15] N. Shavit and D. Touitou. Software transactional memory. In Proc. of the 12th Annual ACM Symposium on Principles of Distributed Computing (PODC), pages 204-213, 1995.

[16] G. Weikum and G. Vossen. Transactional Information Systems: Theory, Algorithms, and the Practice of Concurrency Control and Recovery. Morgan Kaufmann, 2002. 


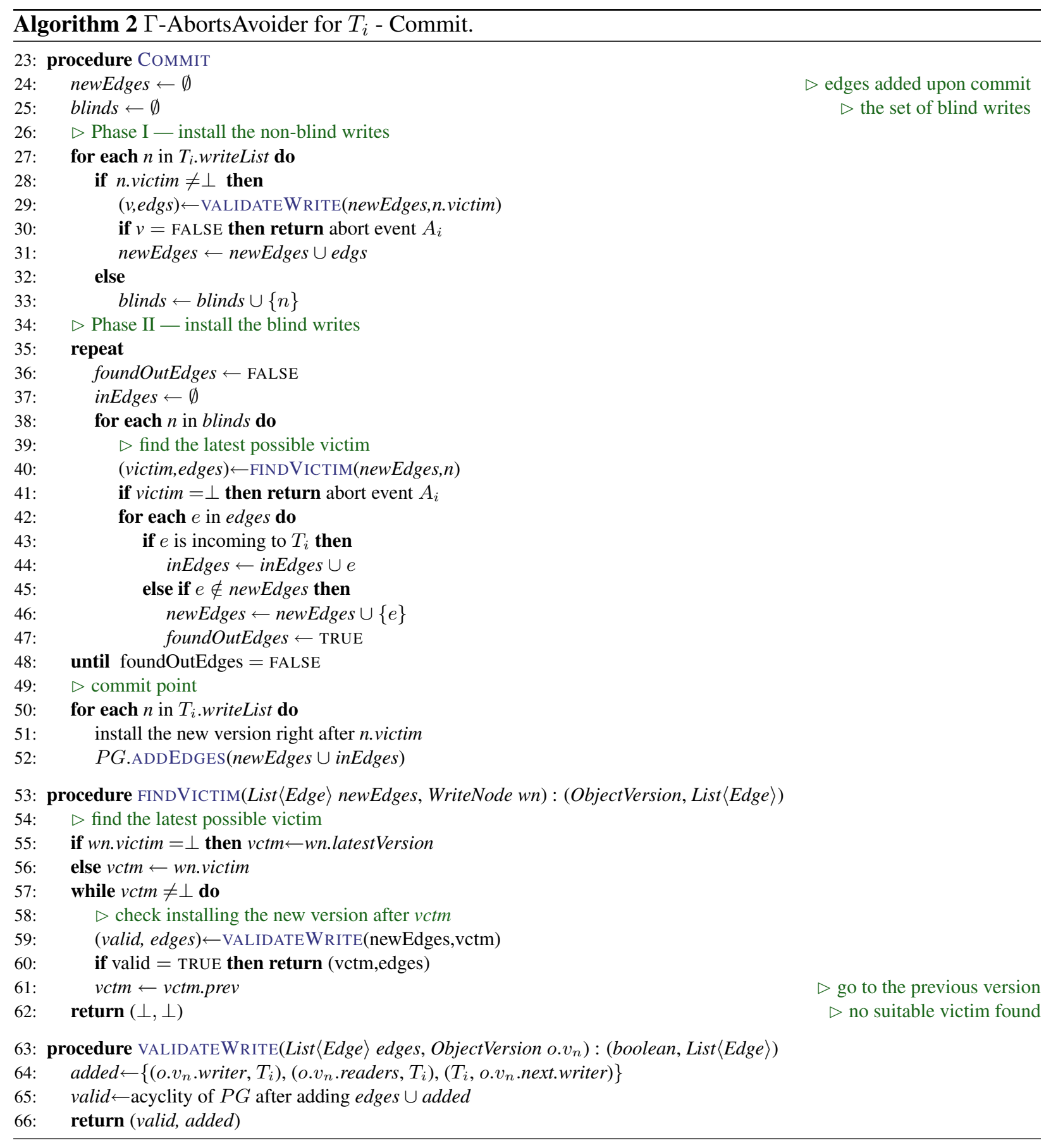

